\title{
IMAGE FUSION BASED ON DTCWT \& PCA IN PRESENCE OF NOISE
}

\author{
A.HARIKA \\ M.Tech, Dept. of ECE, SVUCE, S.V University, Tirupati, India
}

\begin{abstract}
: image fusion is a process of combining relevant information from two or more images into a more informative single image. Image fusion technique improves the quality of the image. In this paper, the fusion is done using dual tree complex wavelet transform and principal component analysis. The images affected by noise are taken as the set of input images. The results show the proposed algorithm has better visual quality. The fusion technique is very much useful in diagnosing and treating cancer in medical fields. This paper is based on fusion of input noisy images using Dual Tree Complex Wavelet Transform and applying Principal Component Analysis (PCA) for fused image such that better image quality is obtained and estimated using various Image quality Metrics. The noise is removed from image and better PSNR values are also obtained.
\end{abstract}

Keywords:Image fusion, Dual tree complex wavelet transform, Principal component analysis, Image quality metrics

\section{I.INTRODUCTION}

Image fusion is defined as a process of combining two or more images into a single composite image. The single output image contains the better scene description than the individual input images. [1] The output image must be more useful for human perception or machine perception. The basic problem of image fusion is determining the best procedure for combining multiple images.

The main aim of image fusion is to improve the quality of information of the output image. The existing fusion algorithms and techniques shows that image fusion provide us an output image with an improved quality.

The requirements for the image fusion process are the fused image should preserve all the relevant information present in the input images and shouldn't introduce any articrafts that may lead to wrong diagnosis.

Image fusion has many applications such as object detection and recognition, medical diagnosis, satellite imaging for remote sensing, military and surveillance, etc. image fusion algorithms can be classified as pixel level and feature level algorithms. The pixel level algorithm directly works on pixels of the input images whereas the feature level algorithms deal with the features extracted from the input images.

\section{II.IMAGE FUSION ALGORITHMS}

Image fusion method can be classified into two types 1. spatial domain fusion methods

2. transform domain fusion method

The spatial domain fusion method deals with the pixels of input images. The fusion methods such as averaging, maximum, minimum, principal component analysis (PCA) and HIS method [1], [2] come under spatial domain approaches.

In the transform domain fusion method, the image is changed to frequency domain. The methods such as dwt, dtcwt etc come under the transform domain or spectral domain.

\section{SPATIAL DOMAIN ALGORITHMS}

\section{1) AvERAGING METHOD}

In this method, the simple average of the pixels is taken in the input images. [1] The mean operation is performed.

Suppose if $A(i, j)$ and $B(i, j)$ are the input images then the fused image $F(i, j)$ can be represented as

$F(i, j)=A(i, j)+B(i, j) / 2$

The main drawback of this method is that both the good and bad information are minimized.

\section{2) MAXIMUM METHOD}

The pixels in both the images are compared and the intensity having more is selected i.e the pixel with maximum value is selected.

Suppose if $A(i, j)$ and $B(i, j)$ are the input images then the fused image $F(i, j)$ can be represented as

$$
\sum_{i=0}^{m} \sum_{j=0}^{\mathrm{n}} \max (A(i, j), B(i, j))
$$

\section{3) MINIMUM METHOD}

In this method the only the minimum pixel values are considered. This is similar to the maximum method. The fused image is obtained by selecting the minimum intensity of the corresponding pixels from both the input images.[2],[3], [7]

Suppose if $A(i, j)$ and $B(i, j)$ are the input images then the fused image $F(i, j)$ can be represented as

$$
\sum_{i=0}^{m} \sum_{\mathrm{j}=0}^{\mathrm{n}} \min (A(i, j), B(i, j))
$$

\section{4) WEIGHTED AVERAGE METHOD}

In this method, the fused image is obtained by taking weighted average intensity of pixels from both the input images.

Suppose if $A(i, j)$ and $B(i, j)$ are the input images then the fused image $F(i, j)$ can be represented as 


$$
\sum_{i=0}^{m} \sum_{\mathrm{j}=0}^{\mathrm{n}} \mathrm{w}(A(i, j))+(1-w) B(i, j)
$$

Where $w$ is the weight factor.

\section{5) PRINCIPAL COMPONENT ANANLYSIS}

PCA [1],[3], [6] method reduces the multi dimensional data sets to lower dimension for analysis. It involves in identifying the patterns in data and expressing the data in such a way as to highlight their similarities and differences. The main advantage of PCA is that after finding the patterns in data, the data can be compressed i.e by reducing the number of dimensions without much loss of data.

Steps involved in PCA are described below:

Step 1: produce the column vectors from input images.

Step 2: calculate the covariance matrix of the column vectors.

Step 3: Calculate the eigen values and the eigen vectors of the covariance matrix.

Step 4: Normalize the column vector

Step 5: normalize the eigen vector values by dividing each element with the mean of eigen vector. These normalize eigen vector values act as the weight values and are multiplied with each pixel of the input image.

Step 6: the sum of two scaled matrices gives the fused image matrix.

\section{6) IHS TRANFORM METHOD:}

The IHS technique [1] is one of the most commonly used fusion techniques for sharpening. It has become a standard procedure in image analysis for color enhancement, feature enhancement, improvement of spatial resolution and the fusion of disparate data sets. In the IHS space, spectral information is mostly reflected on the hue and the saturation. From the visual system, one can conclude that the intensity change has little effect on the spectral information and is easy to deal with. For the fusion of the high-resolution and multispectral remote sensing images, the goal is ensuring the spectral information and adding the detail information of high spatial resolution.

\section{SPECTRAL DOMAIN ALGORITHMS}

The spatial domain methods are having drawbacks like blocking effects, reducing the contrast of the entire image. To overcome the drawbacks we go for spectral domain methods.

In frequency domain methods, the input images are decomposed into multi scale coefficients. Pyramid and wavelet transforms are used as multi resolution filters. There are many approaches such as dwt, curvelet transform method and dual tree complex wavelet transform.etc.

\section{DUAL TREE COMPLEX WAVELET TRANSFORM}

In the dual tree complex wavelet transform method, [1], [2], the fusion is performed using masks to extract information from decomposed structure of DT-
CWT [3]. The DT-CWT $[8,9,10,11,12]$ structure involves both the real and complex coefficients. This complex transform uses two separate DWT decompositions. It is divided into two separate trees, tree $\mathrm{A}$ and tree $\mathrm{B}$ as shown in figure below.

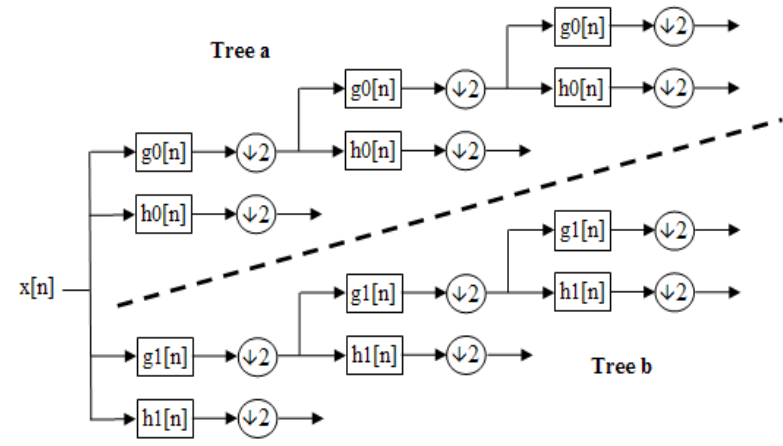

Figure 1: DT-CWT structure

The fusion process involves the formation of the fused pyramid using DT-CWT coefficients which are obtained from the decomposed pyramids of the input source images. The fused image is formed by applying inverse DT-CWT transform.

In figure 2 the fusion process based on DT-CWT is described

DT-CWT preserves the subtle texture regions of brain in MRI images. Ringing effects are reduced and edge details are also retained more clearly. This DT-CWT is more advantageous than the DWT method as it is directional sensitivity and shift variant in nature.

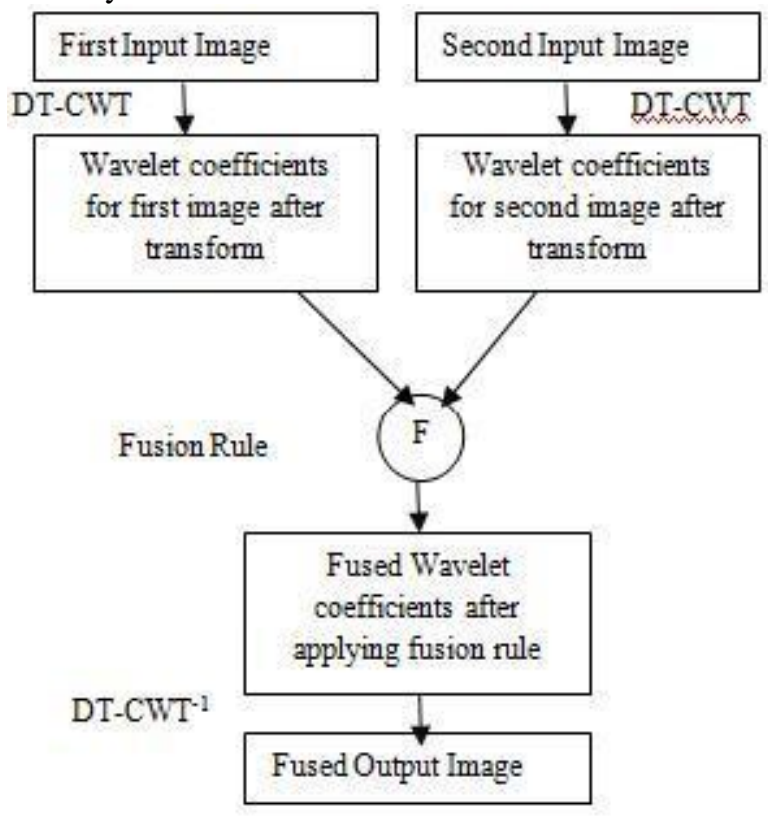

Figure2: DT-CWT based fusion

\section{HYBRID METHOD}

The proposed hybrid method is a combination of the spectral domain and the spatial domain. To overcome the drawbacks of the spectral domain and spatial domain we go for the hybridization. The DT-CWT method in the 
spectral fusion method and PCA method in spatial fusion methods are combined to form a new hybrid method.

The images corrupted by noise are the input images. These input images are decomposed to obtain LL band. Decomposition is performed to form a sequence of pyramids. Low frequency data is present in baseband. Apply masks on the corresponding bands and then mark these filtered bands. The coefficient having the absolute value more at the spatial location is chosen. The PCA method is applied. The noise is removed from the images. The various image quality metrics are analyzed.

Steps involved in hybrid method:

Step 1: The DT-CWT method is applied to each of the input noisy images. Decomposition is performed.

Step 2: the sequence of the pyramids are formed.

Step 3: Apply masks to corresponding bands and mark the filtered bands as and choose the coefficient value such that the value at spatial location is more.

Step 4: apply PCA for the fused image.

Step 5: noise is removed at this stage.

Step6: the image is analyzed by various metrics.

The better quality image is obtained by this process and the output images are free from noise.

The images corrupted by the salt and pepper noise are the input images and this noise is removed by the median filtering. The median filter is an effective tool to remove this type of noise.

\section{IMAGE QUALITY METRICS}

Quality is a characteristic that measures perceived image degradation i.e., in comparison with ideal or perfect image.

Evaluation forms an essential part in the development of image fusion techniques. It involves Full Reference where quality is measured in comparison with ideal image and No Reference Methods, which have no reference image. Here we employ Full reference Methods.

Assumptions made in the following equations are as $\mathrm{A}$ is the image which is perfect, $B$ is the resultant image. $i, j$ is the pixel row and column index.

1. Mean Square Error (MSE)

$$
\text { MSE }=\frac{1}{m n} \sum_{i=0}^{m} \sum_{j=0}^{n}\left(A_{i j}-B_{i j}\right)^{2}
$$

2. Peak Signal to Noise Ratio (PSNR)

$$
\text { PSNR }=10 \times \log _{10}\left(\frac{\text { peak }^{2}}{M S E}\right)
$$

3. Average Difference (AD)

$$
\mathrm{AD}=\frac{1}{m n} \sum_{i=0}^{m} \sum_{j=0}^{n}\left(A_{i j}-B_{i j}\right)
$$

4. Structural Content (SC)

$$
\mathbf{S C}=\frac{\sum_{\mathbf{i} * 0}^{\mathrm{m}} \sum_{\mathbf{j}=\mathbf{0}}^{\mathrm{n}}\left(\mathbf{A}_{\mathrm{ij}}\right)^{2}}{\sum_{\mathbf{i} * 0}^{\mathrm{m}} \sum_{\mathbf{j}=\mathbf{0}}^{\mathrm{n}}\left(\mathbf{B}_{\mathrm{ij}}\right)^{2}}
$$

5. Normalized Cross - Correlation (NCC)

$$
\mathbf{N C C}=\frac{\sum_{\mathbf{i} * \mathbf{0}}^{\mathrm{m}} \sum_{\mathbf{j}=\mathbf{0}}^{\mathrm{n}}\left(\mathbf{A}_{\mathrm{ij}} * \boldsymbol{B}_{i j}\right)}{\sum_{\mathbf{i} * \mathbf{0}}^{\mathbf{m}} \sum_{\mathbf{j}=\mathbf{0}}^{\mathbf{n}}\left(\mathbf{A}_{\mathbf{i j}}\right)^{2}}
$$

6. Maximum Difference (MD)

$\mathbf{M D}=\max \left(\mathrm{A}_{\mathrm{ij}}-\mathrm{B}_{\mathrm{ij}}\right) \mathrm{i}=1.2 \ldots \ldots \mathrm{m}$, $\mathrm{j}=1,2, \ldots . \mathrm{n}$

7. Normalized Absolute Error (NAE)

$$
\mathrm{NCC}=\frac{\sum_{\mathrm{i} * \mathbf{0}}^{\mathrm{m}} \sum_{\mathrm{j}=\mathbf{0}}^{\mathrm{n}}\left(\mathbf{A}_{\mathrm{ij}}-\boldsymbol{B}_{\boldsymbol{i j}}\right)}{\sum_{\mathrm{i} * 0}^{\mathrm{m}} \sum_{\mathrm{j}=\mathbf{0}}^{\mathrm{n}} A_{\mathrm{ij}}}
$$

8. Laplacian Mean Squared Error (LMSE)

$$
\mathrm{LMSE}=\frac{\sum_{i=0}^{m} \sum_{j=0}^{n}(\operatorname{del} 2(A)-\operatorname{del} 2(B))^{2}}{\sum_{i=0}^{m} \sum_{j=0}^{n}(\operatorname{del} 2(A))^{2}}
$$

\section{IV.EXPERIMENTAL RESULTS}

The experiments have been carried out on set of natural images and medical images. Some examples are shown below.

4.1 Example 1:

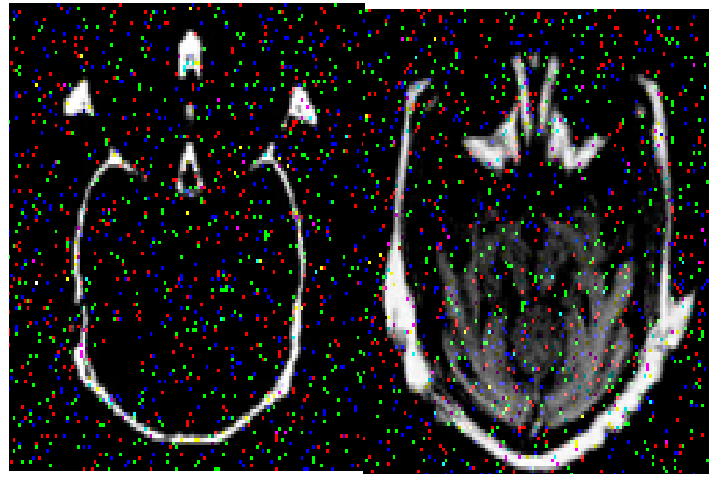

Fig 3: pair of input noisy images.

A CT image and MRI image

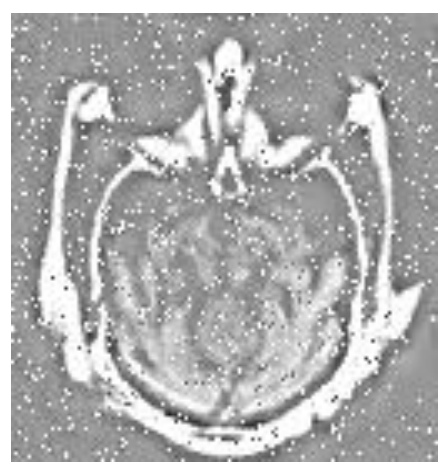

Fig 3.1: fused DT-CWT image

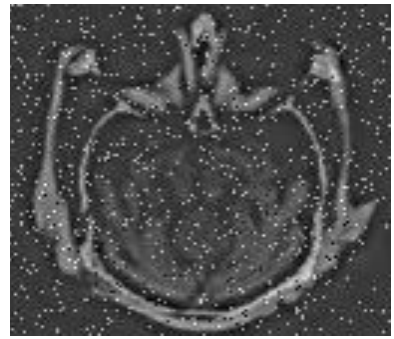

Fig 3.2: fused image using DT-CWT and PCA 
International Journal of Advanced Research in Computer and Communication Engineering Vol. 3, Issue 11, November 2014

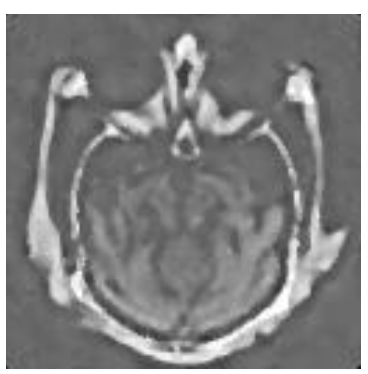

Fig 3.3: filtered output image

4.2 Example 2

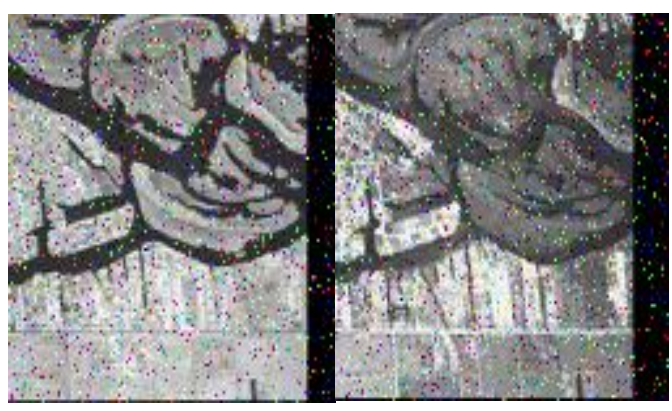

Fig 4: satellite map images of same scene

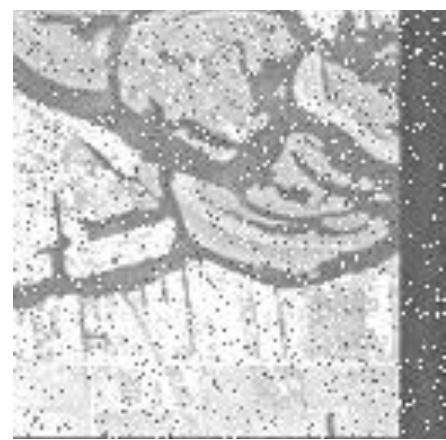

Fig 4.1: fused DT-CWT image

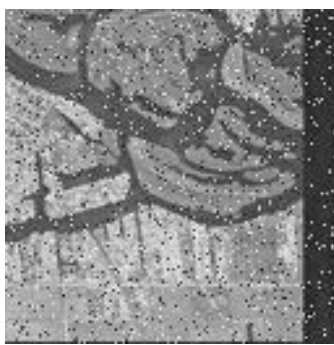

Fig 4.2: fused image using DT-CWT and PCA

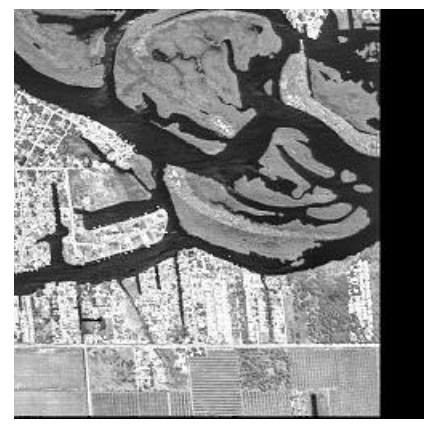

Fig 4.3: filtered image

\begin{tabular}{|l|l|l|}
\hline Image Quality Metrics of example 2 & $\begin{array}{l}\text { Output } \\
\text { of } \\
\text { DTCWT }\end{array}$ & $\begin{array}{l}\text { Output of } \\
\text { DTCWT } \\
\text { and PCA }\end{array}$ \\
\hline Mean square error(MSE) & 0.376 & 0.085 \\
\hline Peak signal to noise ratio(PSNR) & 52.37 & 58.79 \\
\hline Average difference (AD) & 0.59 & 0.27 \\
\hline Structural content (SC) & 0.25 & 0.51 \\
\hline Maximum difference (MD) & 1.72 & 0.77 \\
\hline Palladian mean square error(LMSE) & 6.53 & 0.75 \\
\hline Normalized cross correlation(NCC) & 1.89 & 1.33 \\
\hline Normalized absolute error (NAE) & 1.21 & 0.55 \\
\hline
\end{tabular}

Table I: TABLE OF QUALITY METRICS

From the table of quality metrics for different images it can be concluded that the DT-CWT and PCA based fusion is having better value of PSNR when compared to DT-CWT. The mean square error value is less in this hybrid method. Thus it proves that proposed method performs the best. According to the visual appearance also we can verify the results. The criterion involves the natural appearance, contrast, enhancement of common features. We can say that proposed hybrid method is better than DT-CWT and also performs well in presence of noise. The results say that signal to noise ratio is increased that means the fused image is having higher information and quality is also good.

\section{REFERENCES}

[1] sruthy s ,Dr latha parameswaran ," image fusion technique using DT-CWT", 978-1-4673-7/13/2013 IEEE.

[2] Kingsbury, N. G. 1998a. The dual - tree complex wavelet transform: anew technique for shift invariance and directional filters, proc. 8th IEEE DSP Workshop, Bryce Canyon, UT, USA, paper no. 86.

[3] Fuse tool - An Image Fusion Toolbox for Mat lab 5.x,http://www.metapix.de/toolbox.htm

[4] The Online Resource for Research in Image Fusion www.imagefusion.org

[5] Lindsay I Smith, - A Tutorial on Principal Component Analysis http://www.cs.otago.ac.nz/cosc453/studnent_tutorials/ principal_components.pdf

[6] Zhang Zhong, - Investigations on Image Fusionll, PhD Thesis, University of Lehigh, USA. May1999

[7] Shivsubramani Krishnamoorthy, Soman K. P, -Implementation and Comparative Study of Image Fusion Algorithms\|, International Journal of Computer Applications, Vol. 19, no. 2, Nov. 2010

[8] Mohd. Shahid, Sumana Gupta, - Novel Masks for multimodality image fusion using DT-CWTI, 9th International Conference on Information Fusion, 2006

[9] C. Sydney, Burrus Ramesh, A. Gopinath and Haitao Guo, Introduction to wavelets and wavelets transforms - A primer, Prentice Hall,1998.

[10] M.H Mitchell Image fusion Theories and Applications.

[11] Deepali A.Godse, Dattatraya S. Bormane (2011) "Wavelet based image fusion using pixel based maximum selection rulell International Journal of Engineering Science and Technology (IJEST), Vol. 3 No. 7 July 2011, ISSN : 0975-5462

[12] Susmitha Vekkot, and Pancham Shukla - A Novel Architecture for Wavelet based Image Fusionll. World Academy of Science, Engineering and Technology 572009 